\title{
Diferenciación de Entamoeba histolytica y Entamoeba dispar en muestras de materia fecal por detección de adhesina de E. histolytica mediante ELISA
}

\author{
Claudia E. Guzmán, Myriam C. López, Patricia Reyes, Jorge E. Gómez, \\ Augusto Corredor, Carlos Agudelo \\ Departamento de Salud Pública y Tropical, Facultad de Medicina, Universidad Nacional de Colombia, \\ Bogotá, D.C., Colombia.
}

Las infecciones causadas por el complejo Entamoeba histolytica/Entamoeba dispar son de distribución cosmopolita. E. histolytica es la única especie patógena, pero la diferenciación con $E$. dispar se hace por métodos bioquímicos. Este trabajo tuvo como finalidad detectar rápidamente la adhesina específica de E. histolytica para diferenciarla de $E$. dispar en materia fecal, así como determinar la frecuencia del complejo $E$. histolytica/E. disparen una población rural de Cundinamarca. En el puesto de salud de La Virgen, Quipile, Cundinamarca, se recolectaron 140 muestras de materia fecal de niños y adultos y se analizaron por el método de concentración (formol-éter) para determinar la frecuencia del complejo $E$. histolytica/E. dispar. Las 23 muestras positivas para este complejo $(16,42 \%)$ se valoraron con la metodología de la prueba de ELISA para la detección de adhesina; se obtuvo una frecuencia de 8,69\% (2/23) de E. histolytica. A 19 pacientes positivos para el complejo E. histolytica/E. dispar se les realizó ELISA para la detección de anticuerpos en sangre contra E. histolytica con resultado negativo. Extrapolando los resultados de la detección de adhesina a la población total de este estudio (2/140), se obtuvo una frecuencia de $1,42 \%$ de $E$. histolytica. La ventaja de la prueba de ELISA para la detección de adhesina es su fácil ejecución que permite hacer un diagnóstico rápido para instaurar un manejo adecuado. Se recomienda la realización de estudios de prevalencia a nivel nacional.

Palabras clave: Entamoeba dispar, Entamoeba histolytica, adhesina específica.

Entamoeba histolytica and Entamoeba dispar differentiation in faeces samples by $E$. histolytica adhesin detection through ELISA

Infections caused by the Entamoeba histolytica/Entamoeba dispar species complex have a worldwide distribution. E. histolytica is the only pathogenic species, but the differentiation with $E$. dispar can only be done by biochemical methods. The aim of this work was to detect the $E$. histolytica specific adhesin molecule to differentiate this parasite from $E$. dispar in faeces samples, as well as to determine the prevalence of infection by the $E$. histolytica/E. dispar complex in a rural population from Cundinamarca, Colombia. One hundred and forty fecal samples were collected at the health center of La Virgen, Quipile, Cundinamarca. They were examined by formalin-ether concentration method to determine the prevalence of E. histolytica/E. dispar. Twenty three positive samples (16.42\%) were submitted to an ELISA test for detection of E. histolytica specific adhesin, out of which only 2 $(8.69 \%)$ were found to be positive for $E$. histolytica. Serum samples from 19 patients infected with $E$. histolytica/E. dispar were submitted to an ELISA test for antibodies against $E$. histolytica; all of them were negative. If the prevalence of specific adhesin test had been applied to all the people included in the study, the estimated prevalence of $E$. histolytica infection would be $1.42 \%(2 / 140)$. The advantage of the ELISA test for specific detection of the E. histolytica adhesion molecule is its easiness, which allows a rapid diagnosis in order to provide adequate treatment. This methodology could be recommended for nationwide epidemiological studies to determine the prevalence of $E$. histolytica infection.

Key words: Entamoeba dispar, Entamoeba histolytica, specific adhesin. 
La infección causada por el complejo Entamoeba histolytica/Entamoeba dispar es de distribución cosmopolita. Se calcula que $1 \%$ de las personas infectadas con $E$. histolytica pueden desarrollar patologías potencialmente fatales como la colitis amebiana fulminante o el absceso hepático amebiano $(1,2)$.

En 1925, Emile Brumpt propuso el concepto de que E.histolytica comprendía dos especies morfológicamente idénticas, indiferenciables. Brumpt llamó a una Entamoeba disparno patógena y a la otra Entamoeba dysenteriae, postulada como la especie patógena causante de la amebiasis invasora (3). En 1978, Sargeaunt y colaboradores (4) diferenciaron mediante patrones isoenzimáticos cepas de E. histolytica aisladas de pacientes con manifestaciones clínicas de amebiasis y de portadores asintomáticos, confirmándose que E. histolytica es un complejo constituido por cepas patógenas y no patógenas.

Actualmente, existe en el comercio un estuche de inmunodiagnóstico rápido y de fácil realización para la diferenciación de E. histolytica y E. dispar, por medio de la detección de adhesina específica de E. histolytica en materia fecal (5-10). Lo elabora la casa comercial Techlab, Inc., Blacksburg, Virginia, que utiliza anticuerpos monoclonales específicos para adhesina de E. histolytica $(9,10)$. $\mathrm{La}$ adhesina o lectina de adherencia galactosa$\mathrm{N}$-acetil-D-galactosamina (Gal/GalNac), aglutina células o precipita glicoconjugados y media la resistencia amebiana a la lisis por los componentes terminales del sistema complemento (C5b-9); la adhesina se une a los componentes C8 y C9 para inhibir la lisis de la ameba por el complejo de ataque de membrana (MAC) $(11,12)$. Esta prueba ha sido estandarizada y evaluada por diversos autores. Haque y colaboradores (9) informaron $44(2,2 \%)$ casos de E. histolytica en 2.000 muestras de materia fecal de una población preescolar y escolar de zonas rurales y urbanas de Bangladesh. En 112 muestras de pacientes sintomáticos gastrointes-

\footnotetext{
Correspondencia:

C. Guzmán, ceguzmanr@hotmail.com

Recibido: 28/11/00; aceptado: 20/4/01
}

tinales o con alto riesgo para amibiasis, como homosexuales o viajeros que consultaron en centros médicos de Canadá, Pillai y colaboradores (10) encontraron $3(2,6 \%)$ casos de E. histolytica. Según los datos de la Encuesta Nacional de Morbilidad realizada en Colombia en 1980, la prevalencia de E. histolytica fue de $12 \%$ (13). Como en ese estudio no se hizo la diferenciación del complejo E. histolytica/E. dispar, se propuso la realización de este proyecto cuyo objetivo principal fue diferenciar $E$. histolytica de $E$. dispar, conociéndose que la primera es la única especie patógena.

\section{Materiales y métodos \\ Muestras de materia fecal}

Las muestras de materia fecal provinieron de niños y adultos en un rango de edad entre 4 y 81 años que asistieron al puesto de salud de La Virgen, Quipile (Cundinamarca) y aceptaron participar voluntariamente o a través de autorización de su representante legal, según el Decreto 2737 de 1989, artículo 13 (Código del Menor). Se recolectaron 140 muestras, sin adición de preservativos, las cuales fueron transportadas al laboratorio para ser procesadas el mismo día.

\section{Obtención de las muestras de sangre}

Se tomaron por venopunción 19 muestras de sangre de los individuos que fueron positivos para el complejo E. histolytica/E. dispar en el examen de materia fecal por concentración.

\section{Diagnóstico parasitológico}

Se procesaron las muestras de materia fecal por el método formol-éter $(14,15)$ y se observaron microscópicamente para buscar quistes del complejo E. histolytica/E. dispary otros parásitos. Posteriormente, se determinó la presencia de adhesina en las muestras que fueron positivas.

\section{Detección de adhesina de E. histolytica en materia fecal mediante la técnica ELISA}

Se analizaron 23 muestras de materia fecal del complejo E. histolytica/E. dispar, de las cuales 3 fueron negativas, 20 positivas para parásitos intestinales y las restantes presentaron estos parásitos: Entamoeba hartmanni, Giardia 
duodenalis, Entamoeba coli, Endolimax nana, lodamoeba butschlii, Blastocystis hominis, Ascaris lumbricoides, Chilomastix meslini, Uncinaria sp. y Myxozoa sp. Estas muestras se procesaron utilizando un estuche de inmunodiagnóstico comercial para detección de adhesina de $E$. histolytica en materia fecal $(6-10,16)$, que se leyó en un colorímetro a una densidad óptica de 450 $\mathrm{nm}$ en el Uniskan I.

\section{Cálculos}

Se determinó el valor de la absorbancia de los controles positivo y negativo y las muestras, restando el valor de absorbancia del control negativo al valor de absorbancia de las muestras, considerándose positiva la absorbancia mayor de 0,05 (6-10,16).

\section{Detección de anticuerpos contra E. histolytica por la técnica de ELISA}

Se realizó detección de anticuerpos anti-IgG contra $E$. histolytica en muestras de sangre de los pacientes positivos (17).

\section{Correlación clínica}

A los pacientes cuyo examen coprológico reveló presencia de quistes del complejo E. histolytical E. dispar se les hizo una historia clínica para correlacionar la presencia o ausencia de signos y síntomas compatibles con amibiasis.

\section{Resultados}

En las 140 muestras de materia fecal analizadas por el método de concentración, la frecuencia encontrada para el complejo E. histolytica/E. disparfue de $16,42 \%(23 / 140)$. La frecuencia de E. hartmanni fue de $1,42 \%(2 / 140)$. Las frecuencias encontradas para otros protozoarios y helmintos se encuentran en el cuadro 1. Todos los pacientes del complejo E. histolytica/E. dispar fueron asintomáticos.

\section{Detección de adhesina de E. histolytica en materia fecal mediante ELISA}

En 23 muestras de materia fecal que fueron positivas para el complejo E. histolytica/E. dispar, se obtuvo una frecuencia de $8,69 \%(2 / 23)$ para $E$. histolytica.

\section{Determinación de anticuerpos contra $E$. histolytica por la técnica de ELISA}

Todas las 19 muestras de suero de pacientes con presencia de quistes del complejo E. histolytica/ E. disparfueron negativas para anticuerpos Ig G contra E. histolytica.

\section{Discusión}

La Investigación Nacional de Morbilidad de 1969 (18) informa datos sobre la prevalencia, intensidad y principales características de los parásitos intestinales que se encuentran en Colombia. En una muestra de 5.026 personas, la prevalencia para E. histolytica fue de $25 \%$; sin embargo, no se realizó diferenciación con $E$. hartmanni. Igualmente, no se realizó diferenciación con $E$. dispar, puesto que no es posible hacerla por microscopía óptica y requiere de otras metodologías. Lo anterior indica que la tasa de prevalencia resultante en este estudio es muy alta pero no necesariamente traduce la situación real de E. histolytica.

En los resultados obtenidos por Corredor y colaboradores en la Encuesta Nacional de Morbilidad realizada en 1980, se encontró una prevalencia de $E$. histolytica de $12,1 \%$ para todo el país (13). En esta evaluación tampoco se realizó la diferenciación de E. histolytica/E. dispar, pero a diferencia de la Investigación Nacional de Morbilidad de 1969, sí se hizo la diferenciación del complejo E. histolytica/E. dispar de la $E$. hartmanni mediante la medición de los quistes. En consecuencia, la prevalencia, como era de esperarse, disminuyó aunque continuó presentándose un sobrediagnóstico por E. histolytica. En los datos hallados en el presente estudio, se encontró una frecuencia de 16,42\% (23/140) para el complejo E. histolytica/ E. dispar, resultado similar al obtenido en el Estudio Nacional de Parasitismo Intestinal de 1980 (13).

En cuanto a diferenciación del complejo $E$. histolytica/E. disparpor detección de adhesina de E. histolytica, la frecuencia encontrada en esta investigación fue de $8,69 \%$ en 23 muestras. Extrapolando estos resultados a la población total estudiada, encontramos que la frecuencia corresponde a 1,42\% para E. histolytica. Así 
mismo, se observa una importante reducción de la frecuencia de E. histolytica cuando se comparan los resultados de este trabajo con los de la Encuesta Nacional de Morbilidad de 1980 (13), de $12,1 \%$ a 1,42\%. Este último dato es posible que se aproxime a la real situación de la frecuencia de E. histolytica en Colombia. En la literatura, los estudios realizados por Pillai y colaboradores (10) en 112 muestras de materia fecal de pacientes sintomáticos gastrointestinales, quienes consultaron en centros médicos urbanos de Canadá y que habían estado en zonas subtropicales y tropicales en los dos años anteriores, o que eran hombres homosexuales o habían viajado en los seis meses anteriores, se encontraron $3(2,6 \%)$ casos positivos para $E$. histolytica por detección de adhesina. En el presente trabajo, con esta técnica se encontró una frecuencia de $1,42 \%$ para la población total estudiada (2/140), concordando con los datos de Pillai et al., aunque los pacientes de este estudio fueron asintomáticos. En el estudio realizado por Haque y colaboradores (9) en 2.000 muestras de materia fecal de preescolares y escolares de zonas rurales y urbanas de Bangladesh, se informó de $44(2,2 \%)$ casos de E. histolytica por detección de antígeno, lo que concuerda con los resultados de este estudio y con las características de la población, que también era asintomática.

Los resultados serológicos fueron negativos para E. histolytica, siendo esto lo esperado según los datos de la historia clínica.

Cuadro 1. Frecuencia de parasitos intestinales en 140 muestras de materia fecal.

\begin{tabular}{lcr}
\hline Parásitos & $\begin{array}{c}\mathbf{N}^{\circ} \\
\text { de muestras } \\
\text { positivas }\end{array}$ & $\%$ \\
\hline Complejo E. histolytica/ E. dispar & 23 & 16,42 \\
Giardia lambia & 13 & 9,21 \\
Entamoeba hartmanni & 2 & 1,42 \\
Entamoeba coli & 59 & 42,14 \\
Endolimax nana & 60 & 42,85 \\
lodamoeba butschlii & 27 & 19,28 \\
Ascaris lumbricoides & 15 & 10,71 \\
Blastocystis hominis & 16 & 11,42 \\
Clilomastis mesnilii & 1 & 0,71 \\
Trichuris trichiura & 3 & 2,14 \\
Uncinaria sp. & 3 & 2,14 \\
Myxobulos sp. & 1 & 0,71 \\
\hline
\end{tabular}

El estudio informa un número significativo de casos del complejo E. histolytica/E. dispar por microscopía óptica, en los cuales el tratamiento es innecesario debido a que sólo 2 de 23 pacientes resultaron positivos para E. histolytica. Podemos decir que, con los resultados obtenidos, se hace un aporte importante al diagnóstico de E. histolytica a nivel nacional. Además, se cuenta con un método rápido debido a que el resultado se obtiene a las dos horas de iniciado el proceso, lo cual permite realizar un diagnóstico y manejo rápido y adecuado. Adicionalmente, con la metodología de la detección de adhesina, se tiene la ventaja de disminuir los tratamientos innecesarios, lo cual repercute en reducción de costos y de efectos colaterales de estos medicamentos.

Este trabajo nos muestra la necesidad de realizar un estudio epidemiológico a nivel nacional con el fin de determinar la prevalencia de la E. histolytica, utilizando como antígeno cepas colombianas de E. histolytica para la obtención de los anticuerpos policlonales.

\section{Agradecimientos}

A la comunidad de la población de La Virgen (Cundinamarca), al personal administrativo y técnico del puesto de salud y a los compañeros de la maestría de Infecciones y Salud en el Trópico.

\section{Referencias}

1. Walsh JA. Problems in recognition and diagnosis of amebiasis: estimation of the global magnitude of morbility and mortality. Rev Infect Dis 1986;8:228-38.

2. Graham C. Amoebic disease. Trans Roy Soc Trop Med Hyg 1998;92:361-4.

3. Brumpt E. Etude sommaire de 1 Entamoeba dispar n. sp. Amibe á kystes quadrinuclées, parasite de l'homme. Bull Acad Med (Paris) 1925;94:942-52.

4. Sargeaunt PG, Williams JE, Green JD. The differentiation of invasive and non-invasive Entamoeba histolytica by isoenzyme electrophoresis. Trans Roy Soc Trop Med Hyg 1978;72:519-21.

5. Jackson TFHG. Entamoeba histolytica and Entamoeba dispar are distinct species; clinical, epidemiology, and serologic evidence. Int J Parasitol 1998;28:181-6.

6. Alder P, Wood SJ, Lee YC, Lee RT, Petri WA, Schnaar RL. High affinity binding of the Entamoeba histolytica lectin to polyvalent $\mathrm{N}$-acetylgalactosaminides. J Biol Chem 1995;270:5164-71. 
7. Burch DJ, Li E, Reed S, Jackson TFH, Stanley SL. Isolation of strain-specific Entamoeba histolytica cDNA clon. J Clin Microbiol 1991;27:696-701.

8. Haque R, Neville L, Hahn P, Petri W. Rapid diagnosis of Entamoeba infection by using Entamoeba and Entamoeba histolytica stool antigen detection kits. J Clin Microbiol 1995;33:2558-61.

9. Haque R, Faruque ASG, Hahn P. Entamoeba histolytica and Entamoeba disparinfection in children in Bangladesh. $\mathrm{J}$ Inf Dis 1997;175:734-6.

10. Pillai D, Keystone J, Sheppard D, Maclean D, Macpherson D, Kain K. Entamoeba histolytica and Entamoeba dispar: epidemilogy and comparison of diagnostic methods in a setting of nonendemicity. Clin Infect Dis 1999;29:1315-8.

11. Mann BJ, Dodson JM, Schoroeder JW, Jockhart LA, Clark CG. Structure and function of the galactose/ $\mathrm{N}$-acetyl-D-galactosamine inhibitable adhesin of Entamoeba histolytica. Arch Med Res 1997;28:S168-9.

12. Radvin JL, Petri WA. Entamoeba histolytica (Amebiasis). En: Mandell GL, Bennett JE, Douglas RG, editors. Principles and practice of infectious diseases. New York. Churchill Livingstone; 1991. p.2159-73.
13. Corredor A, Arciniegas E, Hernández CA, Cáceres E, Castaño L, Estupiñan D, et al. Parasitismo intestinal. Santafé de Bogotá, D.C.: Instituto Nacional de Salud; 2000.

14. Allen AVH, Ridley DS. Further observations on the formol-ether concentration technique for fecal parasites. J Clin Pathol 1970;23:545-6.

15. Ridley DS, Hawgood BC. The value of formol-ether concentration of faecal cyst and ova. J Clin Pathol 1956; 9:74-6.

16. González A, Haque R, Rehman T, Aguirre A, Hall A, Guhl F, et al. Diagnosis of amebic disentery by detection of Entamoeba histolytica fecal antigen by an invasive strain-specific, monoclonal antibody-based enzymelinked immunosorbent assay. J Clin Microbiol 1994;32:96470.

17. Nicholls S, Restrepo M, Duque S, López C, Corredor A. Standardization and evaluation of ELISA for the serodiagnosis of amoebic liver abscess. Mem Inst Oswaldo Cruz 1994;89:53-8.

18. Galán R, Agualimpia C, Corredor A, Cáceres E. Parasitismo intestinal. Investigación Nacional de Morbilidad. Bogotá: Instituto Nacional de Salud; 1969. 